Article

\title{
Few Differences in Energy Intake and Diet Quality of Children and Adolescents across BMI Categories, with and without Accounting for Underreporters: NHANES 2005-2014
}

\author{
Rosanna P. Watowicz * (D) and Rosa K. Hand
}

check for updates

Citation: Watowicz, R.P.; Hand, R.K. Few Differences in Energy Intake and Diet Quality of Children and Adolescents across BMI Categories, with and without Accounting for Underreporters: NHANES 2005-2014. Dietetics 2022, 1, 15-24. https:// doi.org/10.3390/dietetics1010003

Received: 14 December 2021

Accepted: 29 January 2022

Published: 4 February 2022

Publisher's Note: MDPI stays neutral with regard to jurisdictional claims in published maps and institutional affiliations.

Copyright: (c) 2022 by the authors. Licensee MDPI, Basel, Switzerland. This article is an open access article distributed under the terms and conditions of the Creative Commons Attribution (CC BY) license (https:/ / creativecommons.org/licenses/by/ $4.0 /)$.

\author{
Department of Nutrition, School of Medicine, Case Western Reserve University, 10900 Euclid Ave., \\ Cleveland, OH 44106, USA; rkh30@case.edu \\ * Correspondence: rpw39@case.edu
}

\begin{abstract}
Previous studies of the relationship between energy intake and child weight have shown mixed results and have largely not described differences in diet quality or the impact of underreporting. The objective of this study was to investigate the relationship between dietary intake and childhood obesity by assessing energy intake and diet quality across BMI categories for 2-19-year-old children before and after excluding underreporters. Energy intake and the Healthy Eating Index 2010 (HEI) were studied for 2-19-year-old children using data from 2005-2014 NHANES. Underreporters were defined as children consuming $<100 \%$ of their predicted resting energy expenditure (REE) and were excluded from some analyses. There were no statistically significant differences in HEI scores across weight categories; mean HEI total score was 47.1 (SE 0.23) out of a possible 100. When considering all 12-19-year-old children, those in the overweight, obesity, and severe obesity categories had a lower mean energy intake than children in the healthy weight category $(p<0.001)$. Excluding underreporters, 6-11-year-old children in the overweight, obesity, and severe obesity categories had a higher energy intake than healthy weight children $(p<0.001)$. In both analyses, children in the overweight, obesity, and severe obesity categories reported consuming a lower \% REE than healthy weight children. Diet quality in all children is low, and the relationship between reported energy intake and BMI remains complex. Healthy eating messages should target all children.
\end{abstract}

Keywords: pediatrics; energy intake; diet quality; obesity; severe obesity; NHANES

\section{Introduction}

The prevalence of childhood obesity in the United States is currently $19.3 \%$ [1], and energy imbalance is widely accepted as a cause of obesity. However, the limitations of dietary assessment make it difficult to test differences in ad libidum energy intake across weight categories. Assessments that measure dietary intake in real time can alter the intake of participants, and assessments that occur at a time after intake rely on memory [2]. Additionally, social desirability bias has been shown to correlate with lower nutrient intake estimation in adults [3]. Therefore, underreporting of dietary intake is a common concern [4] and may be a reason why, to date, studies of the relationship between childhood obesity and energy intake have been mixed in their findings. For example, a systematic review conducted by the Academy of Nutrition and Dietetics showed that, when examining the relationship between energy intake and adiposity in children, some studies found a positive relationship, some a negative relationship, and some found no relationship [5].

Although the relationship between energy intake and obesity has been studied extensively in children, there are three gaps in the literature that this study aims to fill. First, there are few studies that attempt to address the limitations of dietary assessment by excluding those children whose reported energy intakes are likely inaccurate. Secondly, few studies have included a measure of diet quality, which may better reflect the complex relationship between diet and weight. Finally, a category of children with severe obesity has been absent 
from most studies conducted prior to 2013. However, a standardized definition for severe obesity (BMI $\geq 120 \%$ of the BMI at the age- and sex-specific 95th percentile, or BMI $\geq 35$ ) has since been accepted by the wider medical community [6], making the inclusion of this category feasible.

Given these gaps, the aims of this study were to: (1) assess mean energy intake and diet quality across age and weight categories for U.S. children and adolescents, including a category for severe obesity, (2) estimate the frequency of underreporting of energy intake, defined as intake $<100 \%$ estimated resting energy expenditure (REE), across age and weight categories, and (3) explore mean energy intake and diet quality across age and weight categories after excluding those identified as underreporters. We hypothesized that, when all children were included in the analysis, there would be no significant differences in energy intake or diet quality across the different weight categories, but when underreporters were excluded, children with obesity and severe obesity would have a higher energy intake and lower diet quality compared with children with a healthy weight.

\section{Materials and Methods}

For this study, we used cross-sectional data for children ages 2 to 19 years (24 to $<240$ months) from five two-year NHANES cycles spanning years 2005 through 2014. Because nationally representative NHANES data are publicly available and de-identified, this study did not qualify as human subjects research and, therefore, institutional review board approval was not sought. Since the Healthy Eating Index score updates with each release of the Dietary Guidelines for Americans [7] and updated Dietary Guidelines for Americans were released in 2015, we elected to analyze NHANES data from dates prior to 2015.

\subsection{Demographic Data}

The child's age in months at the NHANES in-person mobile examination center exam was used to categorize the children into one of three groups: 2 to 5 years (24-71 months), 6 to 11 years (72-143 months), and 12 to 19 years (144-239 months). The child's gender and race/ethnicity were reported in the NHANES demographic data files.

\subsection{Anthropometric Data}

Height and weight were measured for all children during the NHANES in-person mobile examination center exam following standard procedures [8]. Body mass index (BMI) was calculated for all children who had height and weight measures. The SAS program published by the Centers for Disease Control and Prevention (available at: https: / /www.cdc. gov/nccdphp/dnpao/growthcharts/resources/sas.htm (accessed 2 February 2022) was used to calculate each child's age- and sex-specific BMI percentile, as well as the percent of the 95th percentile for each child's BMI.

Children were categorized into one of five mutually exclusive weight categories based on their BMI: underweight (BMI $<5$ th percentile), healthy weight (BMI $\geq 5$ th percentile and $<85$ th percentile), overweight (BMI $\geq 85$ th percentile and $<95$ th percentile), obesity (BMI $\geq 95$ th percentile but not meeting the criteria for severe obesity), and severe obesity (BMI $\geq 120 \%$ of the BMI at the age- and sex-specific 95th percentile, or BMI $\geq 35$ ). Children missing a BMI value (due to missing either a height or weight measurement) were excluded from analysis.

\subsection{Dietary Data and Healthy Eating Index 2010}

During the NHANES in-person examination, a 24-h dietary recall was collected for all children. The 24-h recall was conducted by a trained researcher using the validated Multiple Pass Method and a measurement guide to help estimate portion sizes $[9,10]$. The previous day's dietary intake was proxy-reported (via parent or caregiver proxy) for children 5 years and younger, proxy-assisted for children 6-11 years, and child-reported for children 12 years and older. We used this 1-day, 24-h recall for all dietary analyses in this study. 
The Healthy Eating Index 2010 (HEI 2010) is a measure of how well one's dietary intake corresponds to the 2010 Dietary Guidelines for Americans [11]. The HEI 2010 includes a total score (possible scores range from 0 to 100, with 100 indicating the best compliance with the Dietary Guidelines), as well as 12 subcomponent scores representing intake of different food categories.

For this study, we used the Food Patterns Equivalents Database files, published by the U.S. Department of Agriculture Food Surveys Research Group, to calculate HEI 2010 scores [12]. The Food Patterns Equivalent Database converts the foods and beverages reported by an individual into 37 different food components (for example, dark green vegetables, whole grains, solid fats). The USDA publishes Food Patterns Equivalent Databases corresponding to the NHANES 24-h dietary recall data for each survey cycle. We used the SAS code, published by the National Cancer Institute Division of Cancer Control \& Population Sciences and available here: https:/ / epi.grants.cancer.gov/hei/sas-code.html (accessed 2 February 2022), along with the Food Patterns Equivalent Database files for each cycle of NHANES to calculate HEI 2010 total and subcomponent scores. HEI 2010 scores were calculated using the simple HEI 2010 scoring algorithm method, which is the recommended method for calculating HEI 2010 scores when using only one 24-h recall and comparing diet quality to another variable (in this case, BMI category) [13].

\subsection{Identifying Underreporters}

We defined underreporters as those children who reported less than $100 \%$ of their estimated resting energy expenditure, as calculated by the Mifflin-St. Jeor equation [14]. The Mifflin-St. Jeor equation has previously been identified as the most accurate predictive equation (when compared with resting energy expenditure measured via indirect calorimetry) for children with severe obesity [15]. Based on conventional knowledge of energy balance and weight, reported energy intake less than estimated energy expenditure would indicate that children either (a) intentionally or unintentionally underreported their energy intake, (b) accurately reported their energy intake but consumed less energy than usual on their day of recall, or (c) accurately reported their energy intake, which aligned with their typical energy intake, but were in a chronic state of negative energy balance leading to weight loss. We acknowledge that reported energy intake of less than 100\% REE may be both plausible and accurate, and the cutoff of $100 \%$ REE is somewhat arbitrary. However, since we were not able to discern which of the above categories each "underreporter" fell into, we grouped all underreporters together and treated these respondents as a separate subsample for certain analyses.

\subsection{Statistical Analysis}

All data were weighted using a 10-year dietary day 1 sample weight, calculated per NHANES analysis guidelines (Day 1 Sample Weight/5) [16]. Complex sampling procedures were used for all analyses, using SDMVSTRA as the strata variable and SDMVPSU as the cluster variable. Children missing energy intake data or a day 1 sample weight, as well as children missing BMI data, were excluded from analysis.

Frequencies were used to analyze demographic data. Mean and standard error were calculated for HEI 2010 total scores, subcomponent scores, and energy intake for each age and weight category for both the total sample and a subsample that excluded underreporters. Frequencies were used to analyze the proportion of underreporters in each age and weight category. One-way ANOVA was used to assess differences in mean HEI 2010 total score and energy intake across weight categories for both the total sample and a subsample that excluded underreporters. Post hoc pairwise comparisons were carried out, using healthy weight children as the comparison group, for variables for which the overall hypothesis test showed statistical significance. To reduce the risk for type I error, we elected not to conduct hypothesis testing for the HEI 2010 subcomponent scores across weight categories. Given the number of hypothesis tests being carried out, we set a conservative a priori significance level of $p<0.01$. SPSS [IBM, version 24] was used for all analyses other 
than calculations of BMI percentile and HEI 2010 score, which were conducted with the SAS software (SAS Institute, Inc., Cary, NC, USA, version 9).

\section{Results}

After excluding 1514 children with missing dietary data (energy intake or dietary sample weight) and 196 children with missing BMI, our final sample included 16,437 children. The demographic characteristics of the sample, mean energy intakes, and HEI 2010 total and subcomponent scores for each age group are listed in Table 1. HEI 2010 total scores decreased in the increasing age groups (Table 1), although statistical significance was not tested.

Table 1. Demographic characteristics and dietary data for 2-19-year-old children, NHANES 2005-2014.

\begin{tabular}{|c|c|c|c|c|c|}
\hline & & 2 to 19 Years & 2 to 5 Years & 6 to 11 Years & 12 to 19 Years \\
\hline & & \multicolumn{4}{|c|}{ \% (Unweighted $n$ ) } \\
\hline & & $100.0 \%(16,437)$ & $21.9 \%(4132)$ & $32.9 \%(5456)$ & $45.1 \%(6849)$ \\
\hline \multirow[t]{2}{*}{ Gender } & Male & $50.9 \%(8365)$ & $51.0 \%(2107)$ & $51.0 \%(2740)$ & $50.9 \%(3518)$ \\
\hline & Female & $49.1 \%(8072)$ & $49.0 \%(2025)$ & $49.0 \%(2716)$ & $49.1 \%(3331)$ \\
\hline \multirow[t]{4}{*}{ Race/Ethnicity } & $\begin{array}{l}\text { Mexican American and } \\
\text { other Hispanic }\end{array}$ & $20.9 \%(5855)$ & $23.6 \%(1519)$ & $21.6 \%(1950)$ & $19.2 \%(2386)$ \\
\hline & Non-Hispanic White & $56.8 \%(4633)$ & $53.5 \%(1205)$ & $56.0 \%(1539)$ & $58.9 \%(1889)$ \\
\hline & Non-Hispanic Black & $14.5 \%(4349)$ & $14.4 \%(978)$ & $14.3 \%(1443)$ & $14.7 \%(1928)$ \\
\hline & $\begin{array}{l}\text { Other race-including } \\
\text { multi-racial }\end{array}$ & $7.8 \%(1600)$ & $8.5 \%(430)$ & $8.1 \%(524)$ & $7.3 \%(646)$ \\
\hline \multirow[t]{6}{*}{ BMI category } & Underweight & $3.5 \%(562)$ & $3.1 \%(164)$ & $3.7 \%(172)$ & $3.5 \%(226)$ \\
\hline & Healthy weight & $64.7 \%(10,309)$ & $73.8 \%(2963)$ & $62.5 \%(3320)$ & $61.9 \%(4026)$ \\
\hline & Overweight & $15.1 \%$ (2539) & $13.2 \%(536)$ & $16.1 \%(864)$ & $15.3 \%(1139)$ \\
\hline & Obesity & $10.9 \%(1964)$ & $8.0 \%(386)$ & $12.4 \%(732)$ & $11.2 \%(846)$ \\
\hline & Severe obesity & $5.9 \%(1063)$ & $1.8 \%(83)$ & $5.3 \%(368)$ & $8.2 \%(612)$ \\
\hline & & \multicolumn{4}{|c|}{ Mean (SE) } \\
\hline Energy (kcal/day) & & $1955(11.4)$ & $1538(11.6)$ & $1936(13.3)$ & 2171 (19.3) \\
\hline \multirow[t]{13}{*}{ HEI 2010} & HEI Total Vegetables & $2.1(0.02)$ & $2.0(0.04)$ & $2.0(0.04)$ & $2.3(0.04)$ \\
\hline & HEI Greens and Beans & $0.67(0.03)$ & $0.66(0.04)$ & $0.66(0.03)$ & $0.68(0.04)$ \\
\hline & HEI Total Fruit & $2.5(0.04)$ & $3.5(0.05)$ & $2.6(0.05)$ & $1.9(0.06)$ \\
\hline & HEI Whole Fruit & $2.3(0.04)$ & $3.0(0.06)$ & $2.5(0.05)$ & $1.8(0.05)$ \\
\hline & HEI Whole Grain & $2.2(0.06)$ & $2.7(0.08)$ & $2.2(0.07)$ & $2.0(0.07)$ \\
\hline & HEI Total Dairy & $6.9(0.05)$ & $8.0(0.07)$ & $7.2(0.07)$ & $6.3(0.08)$ \\
\hline & HEI Total Protein & $3.6(0.03)$ & $3.4(0.04)$ & $3.5(0.04)$ & $3.7(0.04)$ \\
\hline & HEI Seafood and Plant Protein & $1.4(0.03)$ & $1.5(0.05)$ & $1.5(0.05)$ & $1.4(0.05)$ \\
\hline & HEI Fatty Acids & $3.9(0.06)$ & $3.3(0.11)$ & $3.7(0.08)$ & $4.2(0.09)$ \\
\hline & HEI Sodium & $5.0(0.06)$ & $5.8(0.08)$ & $5.1(0.09)$ & $4.5(0.08)$ \\
\hline & HEI Refined Grains & $5.2(0.05)$ & $6.2(0.08)$ & $4.8(0.07)$ & $5.0(0.07)$ \\
\hline & HEI Empty Calories & $11.4(0.10)$ & $12.9(0.13)$ & $11.0(0.14)$ & $10.9(0.14)$ \\
\hline & HEI Total Score & $47.1(0.23)$ & $52.8(0.39)$ & $46.9(0.34)$ & $44.7(0.31)$ \\
\hline
\end{tabular}

Abbreviations: $n$, sample size; NHANES, National Health and Nutrition Examination Survey; BMI, body mass index; HEI 2010, Healthy Eating Index 2010.

Within the age categories, there were no statistical differences in HEI 2010 total score across weight categories (Table 2, panel a). There was a statistically significant difference in energy intake across weight categories for 12-19-year-old children $(p<0.001)$, with post hoc analysis indicating that children in the overweight (2019 kcal/day), obesity (2013 kcal/day), and severe obesity $(1955 \mathrm{kcal} /$ day) categories had significantly lower mean energy intake compared with children in the referent healthy weight category ( $2260 \mathrm{kcal} /$ day $)$. This same pattern was observed for both 6-11-year-olds and 12-19-year-olds when examining energy intake as a percentage of resting energy expenditure (\% REE), which standardizes energy intake for age and body size; those in the overweight, obese, and severe obesity categories reported consuming a significantly lower percentage of their resting energy expenditure. 
Table 2. Energy intake and Healthy Eating Index 2010 scores for 2-19-year-old children.

\begin{tabular}{|c|c|c|c|c|c|c|c|}
\hline \multirow[t]{2}{*}{ a. All Children } & & Underweight & Healthy Weight & Overweight & Obese & $\begin{array}{l}\text { Severe } \\
\text { Obesity }\end{array}$ & $p$-Value \\
\hline & & \multicolumn{5}{|c|}{ Mean (SE) } & \\
\hline \multirow[t]{4}{*}{2 to 5 years } & & $n=164$ & $n=2963$ & $n=536$ & $n=386$ & $n=83$ & \\
\hline & $\%$ REE reported & $232.0(7.2)$ & $222.1(2.0)$ & $212.7(4.6)$ & $207.4(5.4)$ & $173.6(7.5) *$ & $<0.001$ \\
\hline & Energy (kcal) & $1534(44.0)$ & $1528(13.0)$ & $1544(34.0)$ & $1616(46.0)$ & $1567(74.0)$ & 0.51 \\
\hline & HEI Total Score & $50.6(1.6)$ & $53.0(0.40)$ & $53.2(0.90)$ & $52.0(0.90)$ & $49.0(1.7)$ & 0.11 \\
\hline \multirow[t]{4}{*}{6 to 11 years } & & $n=172$ & $n=3320$ & $n=864$ & $n=732$ & $n=368$ & \\
\hline & \% REE reported & $200.0(5.2)$ & $192.4(1.6)$ & $173.7(3.4) *$ & $172.0(3.6)$ * & $142.5(3.9)$ * & $<0.001$ \\
\hline & Energy (kcal) & $1842(56.0)$ & $1913(16.0)$ & 1957 (40.0) & 2047 (40.0) & $1567(74.0)$ & 0.03 \\
\hline & HEI Total Score & $46.3(1.8)$ & $47.0(0.40)$ & $46.7(0.90)$ & $46.6(0.60)$ & $46.8(1.0)$ & 0.98 \\
\hline \multirow[t]{4}{*}{12 to 19 years } & & $n=226$ & $n=4026$ & $n=1139$ & $n=846$ & $n=612$ & \\
\hline & $\%$ REE reported & $170.7(5.2) *$ & $153.9(1.8)$ & $125.8(1.8) *$ & $115.6(2.5) *$ & $99.3(2.7) *$ & $<0.001$ \\
\hline & Energy (kcal) & $2270(78.0)$ & $2260(29.0)$ & $2019(35.0)$ * & $2013(51.0) *$ & $1955(61.0) *$ & $<0.001$ \\
\hline & HEI Total Score & $43.1(1.0)$ & $44.7(0.40)$ & $44.3(0.60)$ & $45.9(0.90)$ & $44.3(0.80)$ & 0.24 \\
\hline \multirow{2}{*}{\multicolumn{2}{|c|}{ b. Underreporters Excluded }} & Underweight & Healthy Weight & Overweight & Obese & $\begin{array}{l}\text { Severe } \\
\text { Obesity }\end{array}$ & $p$-Value \\
\hline & & \multicolumn{6}{|c|}{ Mean (SE) } \\
\hline \multirow[t]{4}{*}{2 to 5 years } & & $n=160$ & $n=2889$ & $n=519$ & $n=374$ & $n=76$ & \\
\hline & $\%$ REE reported & $234.9(7.0)$ & $224.7(2.0)$ & $215.8(4.5)$ & $210.6(5.1)$ & $180.3(8.0) *$ & $<0.001$ \\
\hline & Energy (kcal) & $1554(44.0)$ & $1545(13.0)$ & $1566(33.0)$ & $1641(46.0)$ & $1626(79.0)$ & 0.41 \\
\hline & HEI Total Score & $50.5(1.6)$ & $53.0(0.40)$ & $52.9(0.90)$ & $52.2(1.0)$ & $48.8(1.8)$ & 0.12 \\
\hline \multirow[t]{4}{*}{6 to 11 years } & & $n=164$ & $n=3121$ & $n=778$ & $n=638$ & $n=291$ & \\
\hline & $\%$ REE reported & $202.6(5.5)$ & $198.0(1.5)$ & $181.4(2.9)$ * & $182.6(3.3) *$ & $156.4(3.6) *$ & $<0.001$ \\
\hline & Energy (kcal) & $1863(59.0)$ & $1965(16.0)$ & $2038(38.0)$ & $2163(36.0)$ * & $2119(49.0) *$ & $<0.001$ \\
\hline & HEI Total Score & $46.4(1.8)$ & $47.0(0.40)$ & $47.0(0.90)$ & $46.2(0.70)$ & $45.7(1.1)$ & 0.75 \\
\hline \multirow[t]{4}{*}{12 to 19 years } & & $n=192$ & $n=3233$ & $n=752$ & $n=479$ & $n=271$ & \\
\hline & $\%$ REE reported & $185.1(5.1)$ & $174.2(1.6)$ & $154.0(2.0) *$ & $150.1(2.7) *$ & $137.6(2.8) *$ & $<0.001$ \\
\hline & Energy (kcal) & $2459(77.0)$ & $2566(27.0)$ & $2467(37.0)$ & $2617(54.0)$ & $2703(73.0)$ & 0.02 \\
\hline & HEI Total Score & $43.4(1.1)$ & $44.7(0.40)$ & $43.8(0.70)$ & $44.6(0.90)$ & $43.5(0.90)$ & 0.48 \\
\hline
\end{tabular}

Abbreviations: $n$, sample size; SE, standard error; REE, resting energy expenditure (estimated using the Mifflin-St. Jeor equation); HEI, Healthy Eating Index 2010; * significantly different than healthy weight, $p<0.01$.

Among all children, the average \% REE reported was 172.6\% (SE 0.8\%). The proportion of children defined as underreporters is listed in Table 3. Generally, the proportion of underreporters was lowest in the youngest age category (2-5-year-old children), and the proportion of underreporters increased with increasing adiposity. More than half (56.8\%) of 12-19-year-old children with severe obesity were considered underreporters compared with $7.3 \%$ of $2-5$-year-old children with severe obesity.

Table 3. Percentage of children that reported energy intake that was less than estimated resting energy expenditure.

\begin{tabular}{lrrrr}
\hline & \multicolumn{1}{c}{ Underweight } & Healthy Weight & \multicolumn{1}{c}{ Overweight } & \multicolumn{1}{c}{ Obese } \\
\cline { 2 - 5 } & & & \% (Unweighted $n$ ) \\
\hline 2 to 5 years & $1.8 \%(4)$ & $1.9 \%(74)$ & $2.4 \%(17)$ & $2.6 \%(12)$ \\
6 to 11 years & $2.1 \%(8)$ & $4.9 \%(199)$ & $7.5 \%(86)$ & $10.5 \%(94)$ \\
12 to 19 years & $14.1 \%(34)$ & $21.0 \%(793)$ & $36.0 \%(387)$ & $45.3 \%(367)$ \\
\hline
\end{tabular}

Abbreviations: $n$, sample size.

The extent (magnitude) of underreporting also increased with increasing adiposity in the oldest age group (Figure 1). In 12-19-year-olds, this was statistically significant by ANOVA, specifically when comparing those underreporters with severe obesity $(70.1 \%$ of REE) with underreporters in the referent healthy weight group (77.2\% of REE) $(p=0.001)$. 


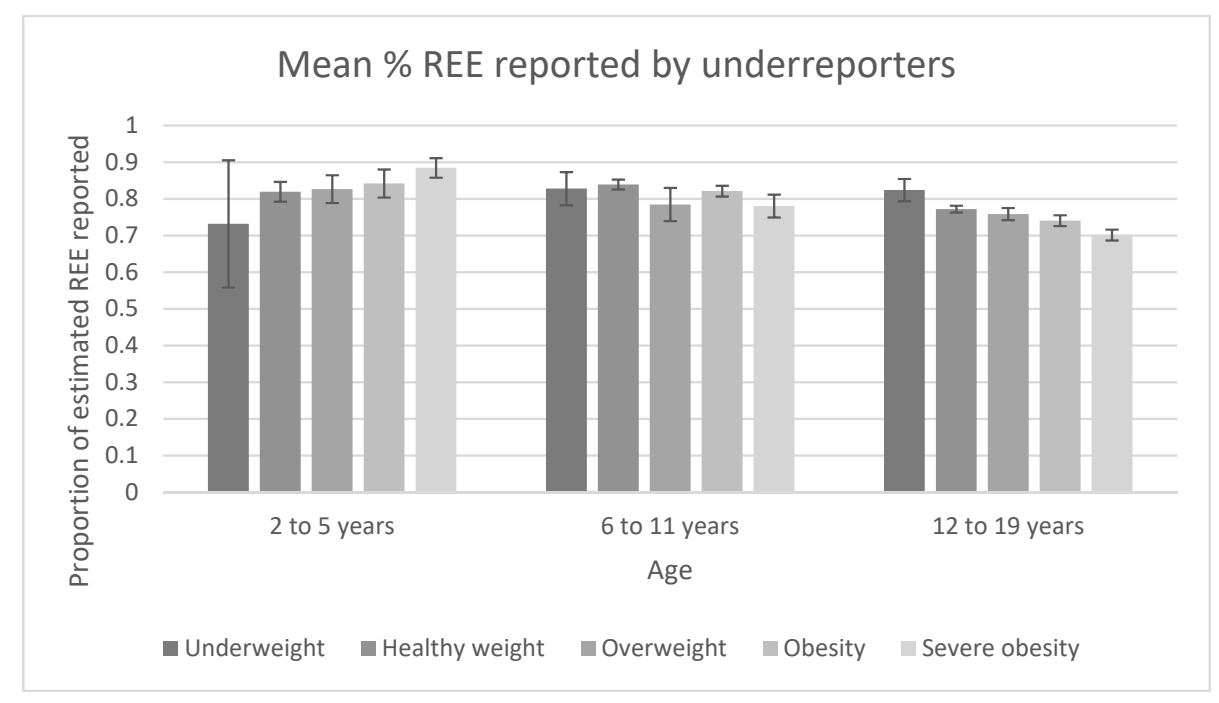

Figure 1. Underreporters were characterized as those children who reported a caloric intake of $<100 \%$ of their estimated REE. Children in the underweight category were the greatest underreporters in the 2-5-year-old age group; dietary intakes for this age group were proxy-reported. They reported the lowest \% REE, and in this age group, the magnitude of underreporting decreased with increasing adiposity. Conversely, children with severe obesity were the greatest underreporters in the 12-19-year-old age group. They reported the lowest \% REE, and in this age group, the magnitude of underreporting increased with increasing adiposity. The extent of underreporting was relatively consistent across all weight categories for 6-11-year-old children.

Mean energy intakes, \% REE reported, and HEI 2010 total and subcomponent scores for the subsample of children that excluded underreporters (subsample $n=13,937$ ) are listed in Table 2, panel b. In this subsample, there were significant differences in raw energy intake across weight categories for $6-11$-year-old children only $(p<0.001)$. Post hoc analysis showed that mean energy intake was significantly higher for children in the obesity ( $2163 \mathrm{kcal} /$ day) and severe obesity ( $2119 \mathrm{kcal} /$ day) categories compared with the reference healthy weight children (1965 kcal/day) category. However, when energy intake was standardized as \% REE, there were significant differences observed within all three age categories; again, in this subsample that excluded underreporters, those in the higher BMI categories reported consuming a lower percent of their resting energy needs (Table 2, panel b). There were no significant differences in HEI 2010 total score observed within any age category.

\section{Discussion}

This study provides evidence that even when we attempt to account for underreporting, a known limitation of dietary assessment, the relationship between energy intake and obesity is still unclear. It is not known whether the "underreporters" in this study were intentionally or unintentionally misreporting their energy intake or if their "underreporting" was accurate and due to an attempt at weight loss. However, this study illustrates that when underreporters are included in the analysis, the relationship between energy intake and obesity is weak.

More specifically, when all children were included in the analysis, there were few significant differences in energy intake across weight categories. The only age group for whom significant differences did exist was for 12-19-year-old children, in which children with overweight and obesity reported consuming significantly fewer calories than healthy weight children, the opposite of what would be expected. Troiano et al. [17] previously conducted a similar analysis of 1988-1994 NHANES data (not excluding underreporters), and they did not identify a significant difference in energy intake for children with a BMI $\geq 95$ th percentile compared with children with a BMI $<$ the 95th percentile. Skinner et al. [18] also 
studied energy intake for children using NHANES data (2001-2008) and found that children with overweight/obesity reported consuming less energy than healthy weight children.

Other studies examining the relationship between energy intake and obesity in children have shown mixed results. In 2007, the Academy of Nutrition and Dietetics conducted a systematic review and found that of 14 longitudinal studies and 29 cross-sectional studies (including Trioano et al.), 5 studies found a positive relationship between obesity and energy intake, 5 studies found mixed results, 25 studies found no significant relationship, and 8 found an inverse relationship between obesity and energy intake [5]. The dietary assessment methods used in these studies varied and included food records, 24-h recalls, and food frequency questionnaires.

When underreporters were excluded from the current analysis, there was a significant difference in reported energy consumption for 6-11-year-old children based on weight category, and the direction of the relationship changed to that which was expected: higher energy intake was associated with the higher weight category. The magnitude of these differences should be noted. For example, among 6-11-year-old children, when underreporters were excluded, children with severe obesity consumed (on average) $150 \mathrm{kcal} /$ day more than children with a healthy weight. This is equivalent to one small snack each day.

Additionally, when reported energy intake was standardized as \% REE, reported intake was lowest for the highest weight categories in all of our analyses, including for the subsample that excluded underreporters. This may be due to the energy required to maintain elevated body weights. For example, the estimated REE for a 64-inch, 125-pound 15 -year-old female is roughly $1350 \mathrm{kcal} /$ day. If this same child is 250 pounds instead, the estimated REE increases to $1916 \mathrm{kcal} /$ day. Therefore, it may simply be more difficult to consume enough calories to reach $200 \%$ REE (for example) for children with higher body weights. Nonetheless, this observation contributes to previous evidence showing that the link between energy intake and weight is unclear.

The higher proportion of underreporters among the higher weight categories is consistent with previous literature $[19,20]$. The reasons for the observed underreporting are unclear. It is likely that for children with obesity, there is a greater risk for social desirability bias leading to underreporting compared with children with a healthy weight. Additionally, children with obesity may be more likely to alter their energy intake in an attempt to lose weight. In this case, the underreporting could be an accurate reflection of their actual intake. Although in this study, excluding underreporters caused the data to better align with our hypothesis, it is important that we do not automatically view these underreporters as unreliable reporters. Future studies could examine this dichotomy by adding an analysis of the NHANES question (asked of children 8 years old and older) regarding attempts to lose weight, as weight loss attempts may be related to diet quantity and quality.

Diet quality was not related to obesity status in any of the analyses conducted in this study. In a systematic review of studies on the relationship between diet quality and obesity in adults, Asghari et al. showed a fairly consistent inverse relationship between BMI and HEI [21]. Studies examining the relationship between obesity and diet quality in children and adolescents are limited. Bekelman et al. found an inverse relationship between HEI 2010 score at age 10 and BMI trajectory over 6 years [22]. Conversely, in a study of 6-year-old children in Tehran, Iran, Askari et al. found that children in the highest tertile of HEI score (using HEI 2015) were more likely to be overweight [23]. Tester et al. examined 1999-2014 NHANES data for 2-5-year-old children and did not observe any significant difference in energy intake or diet quality for children with severe obesity compared with children with a healthy weight [24]. The present study confirms their findings, as we did not see any differences in energy intake or diet quality across weight categories for 2-5-year-old children, even when underreporters were excluded. Notably, 2-5-year-old children had the lowest proportion of underreporters within each weight category.

There are several limitations to this study. These data are cross-sectional and represent only 1 day of intake. This is a particularly important limitation to consider when interpreting HEI 2010 scores; the simple HEI 2010 scoring algorithm method does not adjust for 
measurement error or account for foods that are consumed episodically. For 2-5-year-old and 6-11-year-old children, dietary intakes are proxy-reported and proxy-assisted, respectively, which may lead to errors in reporting. Our cutoff value defining underreporters $(<100 \%$ REE), although based on the assumption that consuming less than their REE would lead to lower adiposity, was somewhat arbitrary. It was also based on estimated (versus measured) REE and did not include a physical activity factor to convert to total energy expenditure. Future research should examine whether a specific cutoff point (\% REE or another indicator) can be used to accurately identify those who underreported their intake.

These data highlight that, among all children in the United States, diet quality is far below the ideal score of 100 (indicating complete alignment with the Dietary Guidelines for Americans) regardless of weight status. From a public health perspective, messages and programs emphasizing healthful eating should be targeted to all children, not just those with overweight or obesity. From a clinical perspective, those working with children with overweight and obesity and their families should be careful not to make assumptions about diet quantity or quality based on weight status but rather conduct individualized dietary assessments. Research is needed to create a validated but practical methodology for conducting dietary assessments in a clinical setting, which is currently lacking [25]. These methods should consider and mitigate underreporting and demonstrate change based on intervention. This may require innovative methods beyond the standard $24-\mathrm{h}$ recall, food record, or food frequency questionnaire and focus on components recommended for assessment based on expert opinion and systematic review: the frequency of eating food outside the home, sugar-sweetened beverage and $100 \%$ juice consumption, portion size, fruit and vegetable intake, intake of high-energy dense foods, number of meals, and quality of snacks [26], along with fat and energy intake [27].

\section{Conclusions}

Overall, these analyses demonstrate ongoing questions about the relationship between energy intake and weight status and should be a call to action for further experimental research regarding these relationships, the role of underreporting, and the third component of energy balance, physical activity. Further, until the relationship between energy intake, diet quality, and weight status is demonstrated consistently in the scientific literature, clinicians and researchers should take care to avoid perpetuating stigmatizing messages about the dietary patterns of children with obesity.

Author Contributions: Conceptualization, R.P.W. and R.K.H.; methodology, R.P.W.; software, R.P.W.; formal analysis, R.P.W. and R.K.H.; investigation, R.P.W. and R.K.H.; resources, R.P.W. and R.K.H.; data curation, R.P.W.; writing—original draft preparation, R.P.W.; writing—review and editing, R.P.W. and R.K.H.; visualization, R.P.W. and R.K.H.; supervision, R.P.W.; project administration, R.P.W.; funding acquisition, not applicable. All authors have read and agreed to the published version of the manuscript.

Funding: This research received no external funding.

Institutional Review Board Statement: Not applicable. This study was a secondary analysis of deidentified data and IRB approval was not required.

Informed Consent Statement: Informed consent was not collected for this study as it was a secondary analysis of publically-available data.

Data Availability Statement: Publicly available datasets were analyzed in this study. These data can be found here: https:/ / wwwn.cdc.gov/nchs/nhanes/Default.aspx (accessed on 2 February 2022).

Acknowledgments: The authors would like to thank Catherine M. Phillips for her assistance in preparing this manuscript for submission.

Conflicts of Interest: Both authors received an honorarium for a presentation at the 2021 Nutrition and Dietetic Educators and Preceptors annual conference. Both authors received an honorarium for a presentation at the 2021 Food \& Nutrition Conference and Expo. The authors have no other conflict of interest to report. 


\section{References}

1. Skinner, A.C.; Ravanbakht, S.N.; Skelton, J.A.; Perrin, E.M.; Armstrong, S.C. Prevalence of Obesity and Severe Obesity in US Children, 1999-2016. Pediatrics 2018, 141, e20173459. [CrossRef]

2. National Cancer Institute. Dietary Assessment Instrument Profiles I Dietary Assessment Primer. Available online: https: / / dietassessmentprimer.cancer.gov/profiles / (accessed on 29 July 2021).

3. Hebert, J.R.; Clemow, L.; Pbert, L.; Ockene, I.S.; Ockene, J.K. Social Desirability Bias in Dietary Self-Report May Compromise the Validity of Dietary Intake Measures. Int. J. Epidemiol. 1995, 24, 389-398. [CrossRef]

4. Freedman, L.S.; Schatzkin, A.; Midthune, D.; Kipnis, V. Dealing With Dietary Measurement Error in Nutritional Cohort Studies. JNCI J. Natl. Cancer Inst. 2011, 103, 1086-1092. [CrossRef]

5. Academy of Nutrition and Dietetics. Evidence Analysis Library. Pediatric Weight Management: Foods and Nutrients. Available online: https: / / www.andeal.org/topic.cfm?cat=4157\&highlight=pwm $\% 3 \mathrm{~A} \% 20$ foods $\% 20 \mathrm{and} \% 20$ nutrients\&home $=1$ (accessed on 20 July 2018).

6. Kelly, A.S.; Barlow, S.E.; Rao, G.; Inge, T.H.; Hayman, L.L.; Steinberger, J.; Urbina, E.M.; Ewing, L.J.; Daniels, S.R. Severe Obesity in Children and Adolescents: Identification, Associated Health Risks, and Treatment Approaches A Scientific Statement from the American Heart Association. Circulation 2013, 128, 1689-1712. [CrossRef] [PubMed]

7. Krebs-Smith, S.M.; Pannucci, T.E.; Subar, A.F.; Kirkpatrick, S.I.; Lerman, J.L.; Tooze, J.A.; Wilson, M.M.; Reedy, J. Update of the Healthy Eating Index: HEI-2015. J. Acad. Nutr. Diet. 2018, 118, 1591-1602. [CrossRef] [PubMed]

8. National Center for Health Statistics. NHANES 2009-2010 Anthropometry Manual. Available online: http:/ / www.cdc.gov/ nchs/nhanes/nhanes2009-2010/current_nhanes_09_10.htm (accessed on 14 April 2014).

9. Blanton, C.A.; Moshfegh, A.J.; Baer, D.J.; Kretsch, M.J. The USDA Automated Multiple-Pass Method accurately estimates group total energy and nutrient intake. J. Nutr. 2006, 136, 2594-2599. [CrossRef] [PubMed]

10. National Center for Health Statistics. NHANES-2013-2014 Survey Operations Manuals. Available online: https:/ /wwwn.cdc gov/nchs/nhanes/continuousnhanes/manuals.aspx?BeginYear=2013 (accessed on 20 July 2018).

11. Center for Nutrition Policy and Promotion. Healthy Eating Index. Available online: http://www.cnpp.usda.gov/ healthyeatingindex (accessed on 4 June 2016).

12. Bowman, S.; Clemens, J.; Thoerig, R.; Friday, J.; Shimizu, M.; Moshfegh, A. Food Patterns Equivalents Database 2009-2020: Methodology and User Guide. 2013. Available online: http://www.ars.usda.gov/main/site_main.htm?modecode=12-35-50-00\# (accessed on 18 October 2013).

13. National Cancer Institute Division of Cancer Control \& Population Sciences. HEI Scores for Examining Association between Diet and Another Variable. National Institutes of Health. Available online: https://epi.grants.cancer.gov/hei/examiningrelationships-between-hei-scores.html (accessed on 4 September 2018).

14. Mifflin, M.D.; St Jeor, S.T.; Hill, L.A.; Scott, B.J.; Daugherty, S.A.; Koh, Y.O. A new predictive equation for resting energy expenditure in healthy individuals. Am. J. Clin. Nutr. 1990, 51, 241-247. [CrossRef] [PubMed]

15. Steinberg, A.; Manlhiot, C.; Cordeiro, K.; Chapman, K.; Pencharz, P.B.; McCrindle, B.W.; Hamilton, J.K. Determining the accuracy of predictive energy expenditure (PREE) equations in severely obese adolescents. Clin. Nutr. Edinb. Scotl. 2017, 36, 1158-1164. [CrossRef] [PubMed]

16. National Center for Health Statistics. National Health and Nutrition Examination Survey Analytic Guidelines, 1999-2010. Available online: http:/ / www.cdc.gov/nchs/data/series/sr_02/sr02_161.pdf (accessed on 27 January 2014).

17. Troiano, R.P.; Briefel, R.R.; Carroll, M.D.; Bialostosky, K. Energy and fat intakes of children and adolescents in the united states: Data from the national health and nutrition examination surveys. Am. J. Clin. Nutr. 2000, 72, 1343S-1353S. [CrossRef] [PubMed]

18. Skinner, A.C.; Steiner, M.J.; Perrin, E.M. Self-reported energy intake by age in overweight and healthy-weight children in NHANES, 2001-2008. Pediatrics 2012, 130, e936-e942. [CrossRef] [PubMed]

19. Bandini, L.G.; Schoeller, D.A.; Cyr, H.N.; Dietz, W.H. Validity of reported energy intake in obese and nonobese adolescents. Am. J. Clin. Nutr. 1990, 52, 421-425. [CrossRef] [PubMed]

20. Waling, M.U.; Larsson, C.L. Energy Intake of Swedish Overweight and Obese Children Is Underestimated Using a Diet History Interview. J. Nutr. 2009, 139, 522-527. [CrossRef] [PubMed]

21. Asghari, G.; Mirmiran, P.; Yuzbashian, E.; Azizi, F. A systematic review of diet quality indices in relation to obesity. Br. J. Nutr. 2017, 117, 1055-1065. [CrossRef] [PubMed]

22. Bekelman, T.A.; Ringham, B.M.; Sauder, K.A.; Johnson, S.L.; Harrall, K.H.; Glueck, D.H.; Dabelea, D. Adherence to index-based dietary patterns in childhood and BMI trajectory during the transition to adolescence: The EPOCH study. Int. J. Obes. 2005 2021, 45, 2439-2446. [CrossRef] [PubMed]

23. Askari, M.; Daneshzad, E.; Naghshi, S.; Bellissimo, N.; Suitor, K.; Azadbakht, L. Healthy eating index and anthropometric status in young children: A cross-sectional study. Clin. Nutr. ESPEN 2021, 45, 306-311. [CrossRef] [PubMed]

24. Tester, J.M.; Phan, T.-L.T.; Tucker, J.M.; Leung, C.W.; Dreyer Gillette, M.L.; Sweeney, B.R.; Kirk, S.; Tindall, A.; Olivo-Marston, S.E.; Eneli, I.U. Characteristics of Children 2 to 5 Years of Age with Severe Obesity. Pediatrics 2018, 141, e20173228. [CrossRef] [PubMed]

25. Krebs, N.F.; Himes, J.H.; Jacobson, D.; Nicklas, T.A.; Guilday, P.; Styne, D. Assessment of Child and Adolescent Overweight and Obesity. Pediatrics 2007, 120 (Suppl. 4), S193-S228. [CrossRef] [PubMed] 
26. Barlow, S.E.; The Expert Committee. Expert Committee Recommendations Regarding the Prevention, Assessment, and Treatment of Child and Adolescent Overweight and Obesity: Summary Report. Pediatrics 2007, 120 (Suppl. 4), S164-S192. [CrossRef] [PubMed]

27. Academy of Nutrition and Dietetics. Evidence Analysis Library Pediatric Weight Management Executive Summary of Recommendations. Available online: https:/ / www.andeal.org/topic.cfm?menu=5296\&pcat=2721\&cat=3013 (accessed on 10 July 2018). 